Archives

6 | 1990

Varia

\title{
Quelques notes sur la forêt ivoirienne
}

\section{Emmanuel Terray}

\section{OpenEdition}

\section{Journals}

Édition électronique

URL : http://journals.openedition.org/ccrh/2869

DOI : $10.4000 /$ ccrh.2869

ISSN : $1760-7906$

\section{Éditeur}

Centre de recherches historiques - EHESS

\section{Édition imprimée}

Date de publication : 15 octobre 1990

ISSN : 0990-9141

\section{Référence électronique}

Emmanuel Terray, "Quelques notes sur la forêt ivoirienne », Les Cahiers du Centre de Recherches Historiques [En ligne], 6 | 1990, mis en ligne le 20 mars 2009, consulté le 10 décembre 2020. URL http://journals.openedition.org/ccrh/2869; DOI : https://doi.org/10.4000/ccrh.2869

Ce document a été généré automatiquement le 10 décembre 2020.

Article L.111-1 du Code de la propriété intellectuelle. 


\title{
Quelques notes sur la forêt ivoirienne
}

\author{
Emmanuel Terray
}

1 Mes enquêtes de terrain m'ont amené à travailler dans deux sociétés forestières: une société lignagère établie au coeur de la silve ivoirienne (les Dida), et une société étatique, située à la limite de la savane et de la forêt (les Abron). Je voudrais donc m'interroger sur la signification et le statut de la forêt pour les divers personnages que l'on rencontre au sein de ces sociétés. J'évoquerai successivement la forêt du cultivateur, celle du chasseur, celle du commerçant, celle du guerrier, et j'examinerai enfin le rôle que les détenteurs du pouvoir de l'Etat font jouer à la forêt.

2 Quelques mots de présentation, tout d'abord : la forêt ivoirienne est une forêt tropicale, pour l'essentiel permanente ; certaines espèces d'arbres perdent leurs feuilles, mais ces chutes se produisent à des époques différentes de l'année, et l'apparence globale de la forêt n'en n'est pas modifiée. Les forêts primaires sont très - et de plus en plus - rares ; nous trouvons donc le plus souvent des forêts secondaires, qui ont été défrichées au moins une fois, et sont caractérisées par des sous-bois très épais. Comme on le sait, on peut distinguer trois niveaux dans la couverture forestière : le plus élevé, celui des grands arbres, atteint 30 à 40 mètres d'altitude; le second, formé par des arbres de taille moyenne, s'élève à 15 ou 20 mètres; au dessous poussent des arbustes, taillis et herbes ; des lianes unissent les trois niveaux. Au sol, la couche d'humus est très mince ; les racines des arbres ne s'enfoncent guère dans la terre, et la stabilité des forêts est assurée par d'impressionnants contreforts. Les niveaux supérieurs sont assez denses pour faire écran à la fois à la lumière du soleil et au souffle du vent ; ils empêchent donc l'évaporation et l'érosion éolienne, et font obstacle à la violence des averses et au ruissellement : l'humus est donc relativement protégé de l'érosion par l'eau.

Pour le villageois, la forêt est d'abord le milieu sur lequel il doit conquérir, année après année, le sol nécessaire à sa vie et à ses cultures. Un village est d'abord une clairière, formée de plusieurs cercles concentriques :

- le territoire même du village ; 
- ses environs immédiats, occupés par des jardins cultivés en permanence, grâce avant tout à l'épandage des excréments humains ;

- le cercle des terres cultivées ou cultivables, où se côtoient parcelles exploitées et jardins.

$\mathrm{Au}$ delà s'étend la forêt intacte, zone réservée aux chasseurs. Seuls les deux premiers cercles sont entièrement et constamment défrichés; le troisième est le théâtre de la «bataille agraire ». Avant l'entrée en scène des cultures industrielles, les peuples de la région pratiquaient en effet l'agriculture itinérante sur brûlis, et elle est encore largement de règle pour les cultures vivrières. On connaît les normes qui régissent cette activité. Plus le temps de jachère est bref, plus faible est la superficie nécessaire, plus réduite est la repousse végétale ; mais en revanche plus compromise est la restauration du sol. Dans la région, on estime que celle-ci est assurée de façon satisfaisante lorsqu'une période de culture de 3 à 4 ans est suivie d'une jachère de 15 à 20 ans; la repousse produit alors, non plus seulement des taillis, mais une véritable forêt secondaire, et le terroir disponible doit être égal à cinq ou six fois la superficie cultivée. Comme il faut environ un hectare de cultures vivrières pour nourrir une famille de cinq personnes, celle-ci doit donc disposer de 5 à 6 hectares au total, l'un cultivé, les autres en jachère.

Des chercheurs britanniques ont essayé d'apprécier, dans la forêt voisine de la forêt du Ghana, la quantité de travail requise par le défrichement de la forêt. Je résume ci-dessous les résultats auxquels ils sont parvenus. Ils ont d'abord tenté d'évaluer le poids de la végétation par acre de forêt : ce poids est de 300 à 700 tonnes dans une forêt primaire, de 40 à 300 tonnes (avant séchage) dans une forêt secondaire; le poids le plus élevé correspond à la forêt secondaire la plus ancienne, le plus réduit à la plus récente. L'opération de défrichement s'accomplit durant les six premiers mois de la saison sèche, d'octobre à mars, et, pour une forêt secondaire récente, elle exige par acre une centaine de journées de travail : 15 jours pour le nettoyage des lianes et des arbustes, 60 jours pour l'abattage des grands arbres; 15 jours pour le brûlage et 10 jours enfin pour le déssouchage. Ce travail doit être effectué tous les trois ans, à l'ouverture de chaque cycle de culture. Pour un hectare, le nombre de journées requises atteint environ 250 ; or pendant la saison sèche, une famille de cinq personnes, dont deux adultes, dispose de 360 journées: on voit donc que l'effort nécessaire, même s'il est intermittent, est cependant très lourd, et on comprend mieux pourquoi l'occupation dense de la forêt sera relativement tardive (au début du second millénaire de notre ère) et attendra la diffusion des outils en fer.

6 La zone des parcelles et des jachères s'étend jusqu'à une circonférence de 6 à 7 kilomètres de rayon au plus. Au delà, les champs seraient trop distants du village, et avant la « paix coloniale», l'insécurité ambiante interdit la construction de campements isolés; on revient chaque soir au village. Plus loin, s'étend la forêt inutilisée, domaine du chasseur.

7 La chasse est l'affaire exclusive des hommes; on peut en distinguer deux formes - la chasse collective, au filet, et la chasse individuelle, à l'arc ou au piège - mais socialement, l'effet est le même: la chasse est l'apanage des jeunes et l'occasion des exploits de l'individu; en effet, les chasses collectives elles-mêmes sont guidées par des leaders, auxquels vont réputation et prestige en cas de succès. La chasse tient une place essentielle dans la subsistance, puisqu'elle est la source quasi-exclusive de l'alimentation carnée; en effet la présence de la mouche tsé tsé interdit l'élevage. Par ailleurs, le chasseur joue un rôle important lors des migrations, puisqu'il connaît les itinéraires, les points d'eau, les zones giboyeuses, etc. Il lui appartient donc de repérer les sites 
favorables pour de nouveaux établissements ; on lui confie également la responsabilité de la délimitation des frontières.

8 Ainsi, le chasseur est un personnage très singulier à l'intérieur de la communauté. Sa valeur individuelle compte pour beaucoup ; les anciens, qui contrôlent la vie collective, se méfient de lui ; comme il vit à la fois dans le monde ordonné et civilisé du village et dans le monde anarchique et sauvage de la forêt, il inspire à ses congénères admiration et peur mêlées; il est toujours suspect d'avoir pactisé avec les puissances obscures de la forêt, toujours craint pour les pouvoirs occultes qu'elles pourraient lui avoir accordés. Un autre personnage occupe une place semblable : l'herboriste, qui parcourt la forêt pour cueillir les «simples». Souvent les deux rôles sont confondus, et l'individu qui les joue est d'autant plus redouté.

9 Au total, à la différence des Pygmées décrits par Turnbull, les horticulteurs regardent la forêt comme un milieu hostile et dangereux, et, à l'exception des chasseurs, ils ne s'y aventurent pas volontiers. On a comparé le village à une île perdue dans l'océan de la forêt, et les chasseurs à de hardis marins prêts à s'éloigner des côtes : l'image est banale, mais elle contient une bonne part de vérité.

10 Au commerçant, la forêt offre des ressources importantes : la noix de kola, dès les temps les plus reculés, et, à la fin du XIXe siècle, le caoutchouc naturel, sans parler de l'or. Mais elle est pour lui une zone difficile d'accès. La mouche tsé tsé et le mauvais état des voies de communication empêchent le recours au portage animal. Toutes les marchandises doivent être chargées à dos - ou plutôt à tête-d'homme. Les prétendues "routes commerciales " sont en réalité de très étroits sentiers, coupés de rivières, de troncs d'arbres déracinés, de fondrières, etc. Le plus souvent les obstacles sont, non pas franchis, mais contournés et l'itinéraire connaît d'interminables méandres, qui allongent considérablement les distances. Au début du XIX siècle, Bowdich estime qu'entre Capecoast et Kumasi on peut parcourir 15 miles par jour, soit les deux tiers de cette distance à vol doiseau. Cette évaluation semble très optimiste; elle est peut-être exacte pour un voyageur, mais elle ne s'applique sûrement pas aux caravanes dont la progression est beaucoup plus lente.

11 Dans les états les mieux organisés, les autorités prennent des mesures pour assurer l'entretien des routes. Dans le royaume Asante, par exemple, les villages sont tenus de défricher les sections qui les traversent, et dès le xvIII ${ }^{e}$ siècle, un corps d'inspecteurs a pour tâche de veiller à l'exécution des travaux; les carences sont frappées d'amendes. En dépit de ces efforts, les commerçants qui animent les réseaux à longue distance - Dyla Hausa - ne pénètreront en forêt que rarement et tardivement. La plupart d'entre eux s'arrêtent dans les cités marchandes qui se trouvent à la lisière de la forêt - Bondoukou, Kintampo, Salaga - et s'approvisionnent auprès d'intermédiaires locaux.

Du point de vue militaire, la forêt constitue un milieu très favorable à la défense, et elle a joué dans l'histoire un rôle essentiel de refuge. En effet, en raison de l'épaisseur des sousbois et des ravages de la mouche tsé tsé, elle est inaccessible aux cavaliers; par ailleurs, elle se prête mal à l'utilisation des armes de jet (flèches, sagaies, frondes). Au contraire, elle est propice aux guet-apens, aux embuscades, aux pièges. En ce qui concerne les armes à feu, elle neutralise la puissance des fusils modernes; en revanche, elle confère une étrange efficacité aux antiques fusils à pierre. Voici comment le lieutenant Largeau, à partir d'une expérience durement acquise au cours de la colonne de Kong, éclaire cet apparent paradoxe : 
Les indigènes de la forêt sont armés de fusils à pierre ; le tir de cette arme est, pour le genre de guerre qu'ils pratiquent, plus efficace que celui du fusil à tir rapide; les combats de l'Akapless et du Baoulé en effet ont toujours été plus meurtriers que ceux qu'on a livrés à Samory.

Cette supériorité du fusil à pierre dans le combat rapproché tient à deux causes : 1 - Etant donné qu'on se bat sous bois à très courte distance, les projectiles contenus dans le fusil (4 ou 5 quelquefois) forment gerbe et atteignent presque toujours au moins un homme, quelquefois deux ou trois. Si d'ailleurs la charge tout entière frappe le même combattant, c'est le plus souvent un homme mort.

2 - Le fusil à pierre bénéficie dans le genre de guerre qui nous occupe de la supériorité des armes à trajectoire courbe sur les armes à trajectoire tendue lorsqu'on se place dans les conditions normales du combat rapproché, c'est à dire quand les hommes visent trop haut parce qu'ils ne visent pas. ${ }^{1}$ des commerçants, à l'invasion des cavaliers, et du même coup, il faut le souligner, à la propagation de l'sslam; mais sous d'autres aspects, elle est un atout et les souverains africains n'ont pas manqué de l'utiliser.

\section{NOTES}

1. Lieutenant Largeau, Notes sur la colonne de Kong, 15 février 1896, Bibliothèque de l'Institut, Fonds Terrier, 5930-2.

\section{AUTEUR}

\section{EMMANUEL TERRAY}

Emmanuel TERRAY est directeur d'études au Centre d'Études Africaines (EHESS). 